\title{
First-principles quasiparticle damping rates in bulk lead
}

\author{
X. Zubizarreta, ${ }^{1,2}$ V. M. Silkin, ${ }^{1,2,3}$ and E. V. Chulkov ${ }^{1,2,4}$ \\ ${ }^{1}$ Donostia International Physics Center, Paseo de Manuel Lardizabal 4, 20018 San Sebastián/Donostia, Basque Country, Spain \\ ${ }^{2}$ Departamento de Física de Materiales, Facultad de Ciencias Químicas, Universidad del País Vasco/Euskal Herriko Unibertsitatea, \\ Apdo. 1072, 20080 San Sebastián/Donostia, Basque Country, Spain \\ ${ }^{3}$ IKERBASQUE, Basque Foundation for Science, 48011 Bilbao, Spain \\ ${ }^{4}$ Centro de Física de Materiales, Centro Mixto CSIC-UPV/EHU, Paseo de Manuel Lardizabal 5, 20018 San Sebastián/Donostia, \\ Basque Country, Spain
}

(Received 18 August 2010; revised manuscript received 28 June 2011; published 26 September 2011)

\begin{abstract}
First-principles calculations of the damping rates (inverse inelastic lifetimes), $\tau^{-1}$, of low-energy quasiparticles in bulk $\mathrm{Pb}$ are presented. Damping rates are obtained both for excited electrons and holes with energies up to $8 \mathrm{eV}$ on a set of $\mathbf{k}$ vectors throughout the Brillouin zone (BZ). Strong localization effects in the calculated $\tau^{-1}$ are found. Averaged over the BZ inelastic lifetimes versus quasiparticle energy are reported as well. Consequences of inclusion of the $a b$ initio $\mathrm{Pb}$ band structure in the lifetime calculation are analyzed. In addition, the effect of the spin-orbit-induced splitting in the band structure on the calculated lifetimes in $\mathrm{Pb}$ is investigated.
\end{abstract}

DOI: $10.1103 /$ PhysRevB.84.115144

PACS number(s): 71.10.Ca

\section{INTRODUCTION}

In metals, electron-electron inelastic scattering processes give rise to the main contribution to the damping rate of excited electrons (and holes) with energies $\gtrsim 0.5 \mathrm{eV}$ above (below) the Fermi level. For a long time, the basic knowledge for such processes was based on free-electrongas (FEG) models $^{1-3}$ or convolution of density of states (DOS). ${ }^{4-6}$ Recently, since the calculations from the first principles have become computationally feasible, this field has experienced profound modifications. Thus, the first-principles calculations $^{7-13}$ have shown that the inelastic lifetime of excited electrons indeed is a result of balance between localization, screening, and band-structure details, even in metals whose electronic structures are frequently considered as being free-electron-like ones. ${ }^{14,15}$ In the previous theoretical work, a non-free-electron-like behavior of damping rates in $\mathrm{Be},{ }^{14,16}$ the role of the screening of the $d$ electrons in inelastic lifetimes in $\mathrm{Cu},{ }^{9,14}$ the transient exciton, ${ }^{17,18}$ and full inclusion of exchange-correlation (XC) effects ${ }^{19}$ as well as renormalization effects on quasiparticle (excited electron or hole) lifetimes in noble and transition paramagnetic ${ }^{20-22}$ and ferromagnetic metals ${ }^{23}$ and compounds ${ }^{24,25}$ were studied by means of the GW approximation. ${ }^{26}$ Using the T-matrix theory, ${ }^{27,28}$ the consequences of spin-flip processes on damping rates in magnetic materials were found to be significant for the spin-minority states. ${ }^{23,29-31}$ In Ref. 32, good agreement was found between the inelastic lifetimes for excited electrons and holes in several metals evaluated within the GW approximation and the semiempirical scattering-theory approach. The role of inclusion of the accurate quasiparticle band structure of $\mathrm{Cu}$ and $\mathrm{Ag}$ in the quasiparticle lifetimes was investigated. . $^{33,34}$

At the same time, in heavy elements an additional ingredient like spin-orbit (SO) interaction starts to be important in the description of the electronic structure. A well-known example is that of corresponding modifications in band structures of $\mathrm{Bi}$ and $\mathrm{Pb}$. However, to the best of our knowledge, up to now the effect of the SO interaction on quasiparticle lifetimes in real materials taking into account its band structure evaluated from first principles was not investigated. In the present paper, for the first time, inelastic lifetimes of excited electrons and holes are studied in bulk lead by means of first-principle calculations, analyzing in detail the band structure as well as SO coupling effects. Recently, thin films of $\mathrm{Pb}$ grown on different substrates have obtained a great deal of attention. The questions regarding growth, transport, magnetic, and superconducting properties of these systems have been considered. For instance, the confinement effects on the superconducting transition temperature ${ }^{35-39}$ and quasiparticle decay rates ${ }^{40,41}$ were studied. Recent lifetime measurements by two-photon spectroscopy $^{41}$ for few-monolayers $\mathrm{Pb}$ deposited on silicon substrate point to the conservation of the bulklike behavior of decay processes in $\mathrm{Pb}$ even in the very thin slabs. The lifetime in $\mathrm{Pb}$ bulk material was investigated with a two-photon photoemission (2PPE) in Ref. 42. In this material, the absence of a momentum-dependent anisotropy in the derived lifetime of the excited electrons with energies in the 2.6-3.2-eV range was observed. At the same time, in a single monolayer of $\mathrm{Pb}$ on $\mathrm{Cu}(111)$, the strong dependence of the lifetime on the momentum was found. ${ }^{42}$ Ultrafast electron dynamics in unoccupied quantum well states (QWSs) in ultrathin $\mathrm{Pb}$ films on $\mathrm{Si}(111)$ has been investigated by femtosecond-timeresolved 2PPE. ${ }^{42,43}$ Lifetime broadening of QWSs has been measured by scanning tunneling spectroscopy in $\mathrm{Pb}$ films on $\operatorname{Ag}(111)^{44}$ and $\mathrm{Cu}(111){ }^{45}$ Therefore, detailed first-principles investigation of the inelastic decay rates of quasiparticles in bulk lead, which can serve as a reference for existing and future experiments, seems useful and timely.

In the present study, the Kohn-Sham equations of densityfunctional theory ${ }^{46,47}$ are solved self-consistently within the norm-conserving pseudopotential scheme. Subsequently, linear-response theory is used to calculate the momentumand energy-dependent density response functions over the Brillouin zone (BZ) from which the imaginary part of the quasiparticle self-energy is computed invoking the GW approximation of many-body theory. Comparison of the obtained results with that for the FEG model shed light on the role of the screening in this metal. In order to study the effect 
of the SO coupling on the quasiparticle lifetime through its influence on the band structure, the latter was evaluated both including and excluding the SO term in the Hamiltonian. The present work demonstrates that the inclusion of the SO interaction into the band-structure calculation produces a noticeable effect in inelastic lifetimes for quasiparticles at very low energies. Nevertheless, the general trends in the quasiparticle inelastic lifetime as a function of its energy are unaffected by the inclusion of the SO splitting. Additionally, comparison of the calculated inelastic lifetimes for the $p$ and $d$ electrons with the same energy reveals strong localization effects.

The paper is organized as follows. In Sec. II, the explicit expressions for the quasiparticle decay rate in periodic crystals within the GW approximation are presented. In Sec. III, the effect of the SO interaction on the electronic band structure of bulk $\mathrm{Pb}$ is discussed. The calculated results for the damping rates are analyzed in Sec. IV along with a link to the linewidth of the QWSs in $\mathrm{Pb}(111)$ thin films. Finally, conclusions are given in Sec. V. Atomic units are used throughout, i.e., $e^{2}=\hbar=m_{e}=1$, unless otherwise stated.

\section{CALCULATION DETAILS}

In the framework of many-body theory, ${ }^{27,28}$ for an inhomogeneous electron system, the damping rate $\tau_{i}^{-1}$ of an excited electron (or hole) in the state $\phi_{i}(\mathbf{r})$ with energy $\varepsilon_{i}$ is obtained from the knowledge of the imaginary part of the quasiparticle self-energy $\Sigma\left(\mathbf{r}, \mathbf{r}^{\prime} ; \varepsilon_{i}\right)$ as

$$
\tau_{i}^{-1}=-2 \int \mathrm{d} \mathbf{r} \int \mathrm{d} \mathbf{r}^{\prime} \phi_{i}^{*}(\mathbf{r}) \operatorname{Im} \Sigma\left(\mathbf{r}, \mathbf{r}^{\prime} ; \varepsilon_{i}\right) \phi_{i}\left(\mathbf{r}^{\prime}\right) .
$$

In order to calculate $\tau^{-1}$ via Eq. (1), the nonlocal energydependent operator $\Sigma\left(\mathbf{r}, \mathbf{r}^{\prime} ; \varepsilon_{i}\right)$ is evaluated by means of the GW approximation, ${ }^{26}$ in which only the first-order term in a series expansion of the self-energy in terms of the screened Coulomb interaction is retained. Moreover, we approximate the self-consistent single-particle Green function $\mathrm{G}$ by that for noninteracting electron system $\mathrm{G}_{0}$, and the screened Coulomb interaction $\mathrm{W}$ is calculated by employing Kohn-Sham wave functions and one-electron energies. This approximation is known to give a reasonably accurate description of the excited electron and hole lifetimes in surface ${ }^{48,49}$ and quantumwell $^{50,51}$ states as well as in bulk states. ${ }^{11}$

For a three-dimensional periodic crystal, an expression for the damping rate of a quasiparticle in an initial state $\phi_{n_{i} \mathbf{k}}(\mathbf{r})$ with energy $\varepsilon_{n_{i} \mathbf{k}}$ takes the form ${ }^{14}$

$$
\begin{aligned}
\tau_{n_{i} \mathbf{k}}^{-1}= & \frac{1}{\pi^{2}} \sum_{n_{f}} \int_{\mathrm{BZ}} \mathrm{d} \mathbf{q} \sum_{\mathbf{G}, \mathbf{G}^{\prime}} \operatorname{Im}\left[-\epsilon_{\mathbf{G}, \mathbf{G}^{\prime}}^{-1}(\mathbf{q}, \omega)\right] \\
& \times \frac{B_{n_{i} n_{f}}^{*}(\mathbf{k}, \mathbf{q}, \mathbf{G}) B_{n_{i} n_{f}}\left(\mathbf{k}, \mathbf{q}, \mathbf{G}^{\prime}\right)}{|\mathbf{q}+\mathbf{G}|^{2}},
\end{aligned}
$$

where the sum is over all final states $\phi_{n_{f} \mathbf{k}}(\mathbf{r})$ with energies $\varepsilon_{n_{f} \mathbf{k}}$ between $\varepsilon_{n_{i} \mathbf{k}}$ and the Fermi level $E_{F}, \omega=\varepsilon_{n_{i} \mathbf{k}}-\varepsilon_{n_{f} \mathbf{k}-\mathbf{q}}$, $\mathbf{G}$ and $\mathbf{G}^{\prime}$ are reciprocal lattice vectors, and

$$
B_{n_{i} n_{f}}(\mathbf{k}, \mathbf{q}, \mathbf{G})=\int d \mathbf{r} \phi_{n_{i} \mathbf{k}}^{*}(\mathbf{r}) e^{\mathrm{i}(\mathbf{q}+\mathbf{G}) \cdot \mathbf{r}} \phi_{n_{f} \mathbf{k}-\mathbf{q}}(\mathbf{r}) .
$$

In Eq. (2), $\epsilon_{\mathbf{G}, \mathbf{G}^{\prime}}^{-1}(\mathbf{q}, \omega)$ are the Fourier coefficients of the inverse dielectric function

$$
\epsilon_{\mathbf{G}, \mathbf{G}^{\prime}}^{-1}(\mathbf{q}, \omega)=\delta_{\mathbf{G}, \mathbf{G}^{\prime}}+\chi_{\mathbf{G}, \mathbf{G}^{\prime}}(\mathbf{q}, \omega) v_{\mathbf{G}^{\prime}}(\mathbf{q}) .
$$

Here, $v_{\mathbf{G}}(\mathbf{q})=4 \pi /|\mathbf{G}+\mathbf{q}|^{2}$ is the Fourier transform of the bare Coulomb potential $v(\mathbf{r})$. In the framework of time-dependent density-functional theory ${ }^{52,53}$ and within the random-phase approximation (RPA), the density response function $\chi$ satisfies the matrix equation

$$
\begin{aligned}
\chi_{\mathbf{G}, \mathbf{G}^{\prime}}(\mathbf{q}, \omega)= & \chi_{\mathbf{G}, \mathbf{G}^{\prime}}^{\mathrm{o}}(\mathbf{q}, \omega)+\sum_{\mathbf{G}^{\prime \prime}} \sum_{\mathbf{G}^{\prime \prime \prime}} \chi_{\mathbf{G}_{\mathbf{G}, \mathbf{G}^{\prime \prime}}^{\mathrm{o}}}(\mathbf{q}, \omega) \\
& \times v_{\mathbf{G}^{\prime \prime}}(\mathbf{q}) \chi_{\mathbf{G}^{\prime \prime \prime}, \mathbf{G}^{\prime}}(\mathbf{q}, \omega),
\end{aligned}
$$

where $\chi_{\mathbf{G}, \mathbf{G}^{\prime}}^{\mathrm{o}}(\mathbf{q}, \omega)$ is the density response function of the noninteracting electrons. Choosing the RPA, one neglects the short-range $\mathrm{XC}$ effects in the evaluation of $\chi$. Matrix $\chi_{\mathbf{G}, \mathbf{G}^{\prime}}^{\mathrm{o}}(\mathbf{q}, \omega)$ reads

$$
\chi_{\mathbf{G}, \mathbf{G}^{\prime}}^{\mathrm{o}}(\mathbf{q}, \omega)=\frac{2}{\Omega} \sum_{\mathbf{k}}^{\mathrm{BZ}} \sum_{n, n^{\prime}} \frac{\left(f_{n \mathbf{k}}-f_{n^{\prime} \mathbf{k}+\mathbf{q}}\right)\left\langle\phi_{n \mathbf{k}}\left|e^{-\mathrm{i}(\mathbf{q}+\mathbf{G}) \cdot \mathbf{r}}\right| \phi_{n^{\prime} \mathbf{k}+\mathbf{q}}\right\rangle\left\langle\phi_{n^{\prime} \mathbf{k}+\mathbf{q}}\left|e^{\mathrm{i}\left(\mathbf{q}+\mathbf{G}^{\prime}\right) \cdot \mathbf{r}}\right| \phi_{n \mathbf{k}}\right\rangle}{\varepsilon_{n \mathbf{k}}-\varepsilon_{n^{\prime} \mathbf{k}+\mathbf{q}}+(\omega+\mathrm{i} \eta)} .
$$

Here, 2 accounts for spin, $\Omega$ is a normalization volume, $f_{n \mathbf{k}}$ is the Fermi distribution function, and $\eta$ is a positive infinitesimal. Note that all matrix elements of the inverse dielectric function $\epsilon_{\mathbf{G}, \mathbf{G}^{\prime}}^{-1}(\mathbf{q}, \omega)$ enter Eq. (2) because of the coupling between wave vectors $\mathbf{q}+\mathbf{G}$ and $\mathbf{q}+\mathbf{G}^{\prime}$ with $\mathbf{G} \neq$ $\mathbf{G}^{\prime}$ as a consequence of the electron-density variation in solids. These coupling terms represent so-called crystalline local-field effects. 54

For the evaluation of the density response function $\chi_{\mathbf{G}, \mathbf{G}^{\prime}}^{\mathbf{o}}(\mathbf{q}, \omega)$ and the matrices $B_{n_{i} n_{f}}(\mathbf{k}, \mathbf{q}, \mathbf{G})$, the eigenfunctions of a Kohn-Sham system without SO interaction are expanded in a plane-wave basis:

$$
\phi_{n \mathbf{k}}(\mathbf{r})=\frac{1}{\sqrt{\Omega}} \sum_{\mathbf{G}} u_{n \mathbf{k}}(\mathbf{G}) e^{\mathrm{i}(\mathbf{q}+\mathbf{G}) \cdot \mathbf{r}} .
$$

When the SO interaction is included into the Kohn-Sham Hamiltonian, one has to work with spinors as eigenfunctions. In this case,

$$
\Phi_{n \mathbf{k}}(\mathbf{r})=\frac{1}{\sqrt{\Omega}} \sum_{\sigma} \sum_{\mathbf{G}} u_{n \sigma \mathbf{k}}(\mathbf{G}) e^{\mathrm{i}(\mathbf{q}+\mathbf{G}) \cdot \mathbf{r}} \xi_{\sigma},
$$

where $\xi_{\uparrow}=\frac{1}{\sqrt{2}}\left(\begin{array}{l}1 \\ 0\end{array}\right)$ for spin up and $\xi_{\downarrow}=\frac{1}{\sqrt{2}}\left(\begin{array}{l}0 \\ 1\end{array}\right)$ for spin down. 


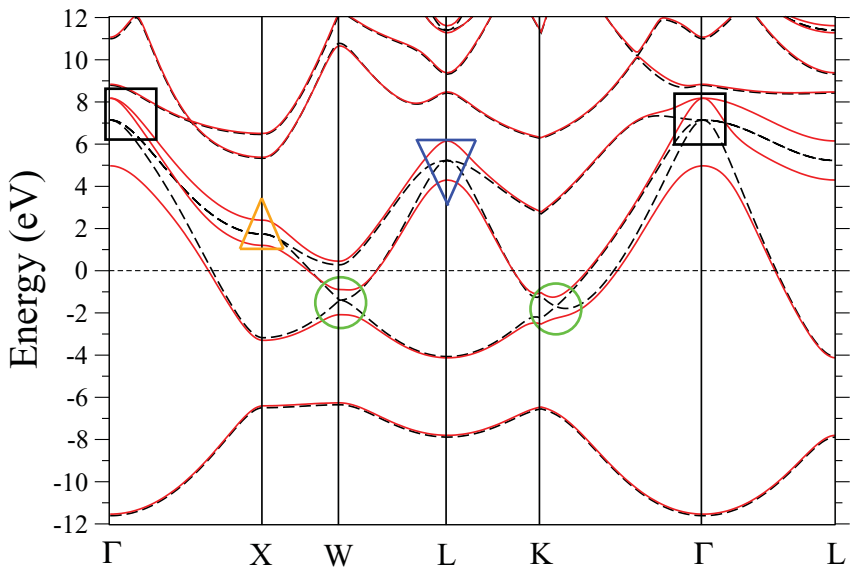

FIG. 1. (Color online) Calculated band structure of bulk lead, with (solid lines) and without (dashed lines) inclusion of the spin-orbit coupling. Symbols mark regions where the SO-induced band splitting is reflected in the density of states shown in Fig. 2. The horizontal dotted line represents the Fermi level.

In this work, the electron-ion interaction was represented by norm-conserving nonlocal pseudopotential, ${ }^{55}$ and the localdensity approximation was chosen for the exchange and correlation potentials in the Ceperley-Alder form ${ }^{56}$ using the Perdew-Zunger parameterization. ${ }^{57}$ Results for the band structure have been obtained with a kinetic-energy cutoff of $14 \mathrm{Ry}$, which corresponds to the inclusion of $\sim 180$ plane waves in the expansion of the Bloch states, guaranteeing energy bands converged to within $20 \mathrm{meV}$.

The inverse dielectric matrices were calculated through Eq. (4) using a Monkhorst Pack $24 \times 24 \times 24$ grid of $\mathbf{q}$ momentum-transfer vectors, for a total amount of $1504 \mathbf{q}$ in the irreducible part of the BZ (IBZ). The evaluation of $\chi^{\circ}$ in Eq. (6) was carried out using a finer $144 \times 144 \times 144$ k sampling with $\approx 32000$ vectors in the IBZ. Also, 25 energy bands were included in calculating $\chi^{\circ}$, spanning energies up to $38 \mathrm{eV}$ above the Fermi level. The broadening parameter $\eta$, employed in the evaluation of $\chi^{0}$ as explained, e.g., in Refs. 58 and 59, was set to $10 \mathrm{meV}$. In the expansion of dielectric matrices, 40 plane waves have been considered. The sums over reciprocal vectors $\mathbf{G}$ and $\mathbf{G}^{\prime}$ in Eq. (2) have been extended over 40 vectors as well.

The main convergence parameter in the calculation of the damping rates is the size of the inverse dielectric matrices used in the evaluation of Eq. (2). The truncation of $\operatorname{Im}\left[-\epsilon_{\mathbf{G}_{,}, \mathbf{G}^{\prime}}^{-1}(\mathbf{q}, \omega)\right]$ affects the value of $\tau_{i}^{-1}$ differently for each $\mathbf{k}$. In practice, the convergence of the damping rates with the size of the inverse dielectric matrices is found to be monotonic, allowing an empirical estimation of the error in the calculated $\tau_{i}^{-1}$ of $\approx 5 \%$ in the most pathological cases.

\section{SPIN-ORBIT EFFECTS IN PB BAND STRUCTURE}

Bulk $\mathrm{Pb}$ lattice has a face-centered-cubic (fcc) crystal structure. Figure 1 presents the calculated band structure for lead along the high-symmetry directions of the BZ obtained with the use of the experimental lattice parameter $a_{c}=4.95 \AA$. In this figure, one set of data (solid lines) corresponds to the calculation with the inclusion of the SO interaction in the

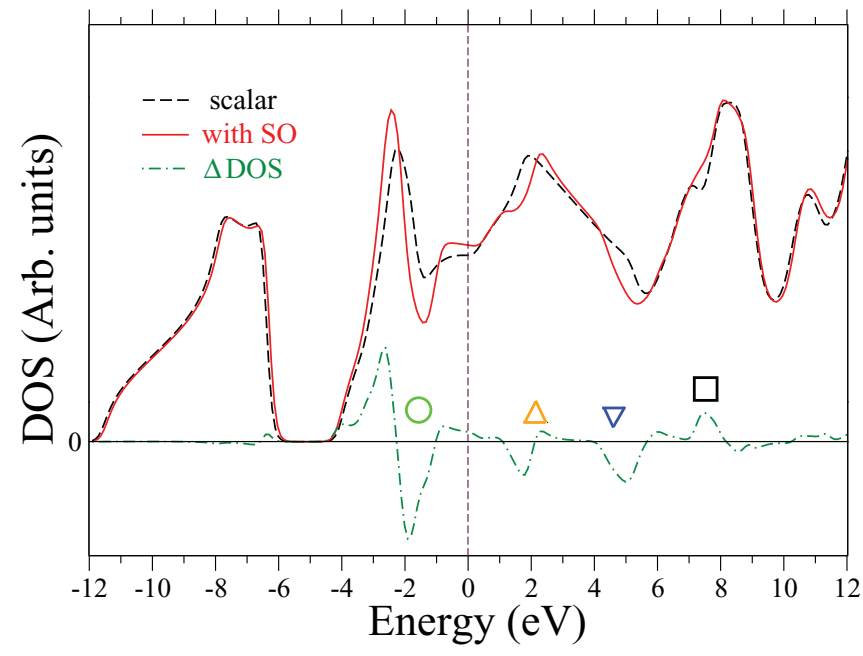

FIG. 2. (Color online) Total density of states (DOS) obtained in the scalar-relativistic calculation $\left(\mathrm{DOS}_{\mathrm{SC}}\right.$, dashed line) and the calculation with inclusion of the spin-orbit (SO) term $\left(\mathrm{DOS}_{\mathrm{SO}}\right.$, solid line). The main variations in the DOS upon the SO inclusion reflected in differential $\triangle \mathrm{DOS}=\mathrm{DOS}_{\mathrm{SO}}-\mathrm{DOS}_{\mathrm{SC}}$ (dashed-dotted line) are related to different band-structure splitting marked by the same symbols as in Fig. 1. DOS is in arbitrary units and energy is according to the Fermi level.

Kohn-Sham Hamiltonian, whereas dashed lines present results with the exclusion of the SO interaction. As the fcc lattice has inversion symmetry, due to the Kramers degeneracy, ${ }^{60}$ each energy band is at least double degenerate in spin in both cases. The calculated band structure is in good agreement with other theoretical results ${ }^{61}$ and with the experimental data ${ }^{62}$ when the SO term is taken into account. As can be seen in Fig. 1, the inclusion of the SO interaction affects only three $p$ energy bands (the only ones that cross the Fermi level), mainly around the high-symmetry points. One can see that the SO interaction produces two main effects in the $\mathrm{Pb}$ band structure:

(a) As the SO coupling breaks the three-dimensional symmetry setting a preferential direction, its inclusion leads to a breaking of symmetry degeneracies at high-symmetry points and directions of the BZ. The most important ones in the case of bulk $\mathrm{Pb}$ are the SO-split $p$ electron bands along $\Gamma X$ and $\Gamma L$ symmetry directions and the energy splitting $\Delta \varepsilon^{\mathrm{SO}}$ at the high-symmetry points: $\Delta \varepsilon^{\mathrm{SO}}=3.21 \mathrm{eV}$ at $\Gamma, 1.20 \mathrm{eV}$ at $X$ and $W$, and $1.85 \mathrm{eV}$ at $L . K$ is the only high-symmetry point where all three $p$-like states are nondegenerate even at the scalar-relativistic level.

(b) The SO interaction leads to the avoiding band-crossing effect, as can be observed between $p$-like states marked by circles in Fig. 1.

In Fig. 2, the total DOS is plotted as a function of energy. The most important effect of inclusion of the SO interaction is the appearance of a valley at $-1.4 \mathrm{eV}$ and the amplitude increase at $-2.4 \mathrm{eV}$ of the peak, whose position is also slightly shifted to higher binding energies. These variations reflect the disappearance of the band-crossing points mentioned above. The other effect of the SO interaction on the band structure mentioned above-breaking of the band degeneracies-has no such important effect on the DOS, because it does not flatten significantly the band dispersion. Nevertheless, the 


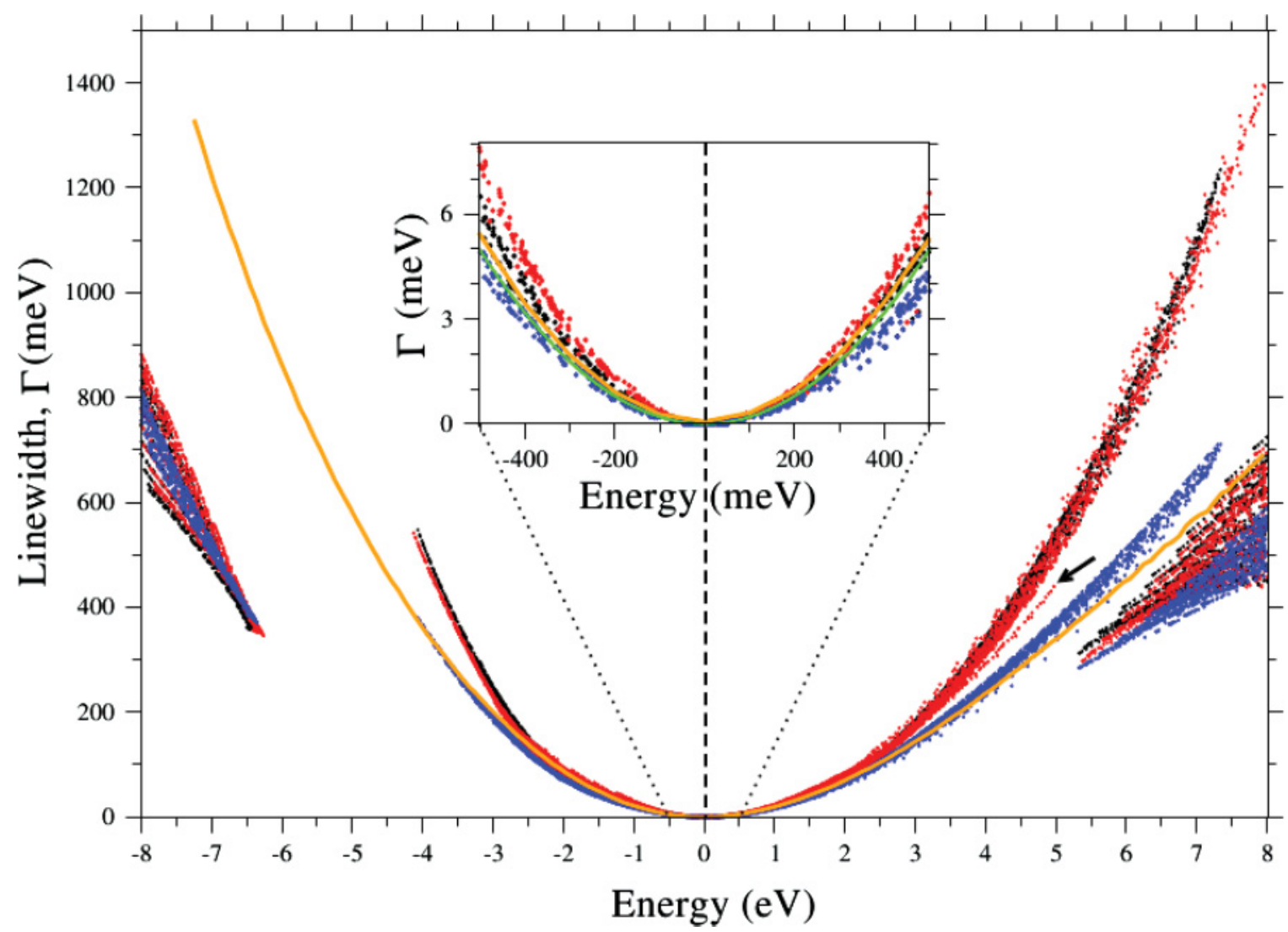

FIG. 3. (Color) Calculated damping rate for hot electrons and holes in all the bands and $\mathbf{k}$ points set over the irreducible part of the Brillouin zone. Red dots represent the values calculated including the spin-orbit coupling in the band structure, the black dots represent the purely scalar-relativistic (SC) results, and the blue dots are the results obtained using the Lindhard dielectric function together with the scalar-relativistic band structure (LDF-SC). The orange line shows linewidth obtained from a free-electron-gas model with the Pb valence charge-density parameter $r_{s}=2.298$ a.u. The green curve in the inset represents results for linewidth calculated according to the Quinn-Ferrel expression of Eq. (9) for the same $r_{s}$. The black arrow points to the linewidth results for the lowest $p$ band near the BZ center calculated with the inclusion of the SO coupling term (see text for more details).

SO-induced splitting around the $X, L$, and $\Gamma$ points affects notably the DOS at $\sim 1.6, \sim 5$, and $\sim 8 \mathrm{eV}$, respectively, as can be seen in Fig. 2 (features in $\triangle \mathrm{DOS}$ are marked by symbols; see also Fig. 1).

In the band-structure calculations, the Hamiltonian including the SO term was solved fully self consistently. However, in the lifetime calculations, the usage of the relativistic wave functions [Eq. (8)] would dramatically increase computational requirements in comparison with the already computationally demanding scalar-relativistic case. Using the relativistic wave functions [Eq. (8)] would demand doubling of the number of plane waves in the basis. As a consequence, a double number of coefficients $u_{n \sigma \mathbf{k}}$ [see Eq. (8)] should be stored. Indeed, as spin-orbit coupling lifts some of the symmetries of the Hamiltonian, $u_{n \sigma \mathbf{k}}$ become complex. Thus, in using the relativistic wave functions one would need roughly four times the memory required for the scalar-relativistic case. Obviously, the computational time would be considerably increased too, especially in the evaluation of $\chi^{0}$, which is one of the most time-consuming steps in the calculation of the damping rates. Therefore, in the SO case as well, in the corresponding calculations the wave functions in Eqs. (6) and (3) were used in the scalar-relativistic form of Eq. (7). In this case, the SO coupling enters the calculations of damping rates through the one-electron energy parts of Eqs. (6) and (2) only. As a result, in the SO case the energy-loss function $\operatorname{Im}\left[-\epsilon_{\mathbf{G}, \mathbf{G}^{\prime}}^{-1}(\mathbf{q}, \omega)\right]$ entering Eq. (2) for the $\tau_{n_{i} \mathbf{k}}^{-1}$ evaluation differs from that used in purely scalar-relativistic calculations. Since the SO-split bulk band structure of $\mathrm{Pb}$, which possesses inversion symmetry, is reproduced in the first-order perturbation theory, ${ }^{63}$ we expect a very weak modification of the scalar relativistic wave function by SO coupling. Therefore, we do not expect that the replacement of the spinor wave function by a scalar one should significantly modify the evaluated SO damping rates. Nevertheless, it would be interesting to quantify its impact in a future work.

\section{DAMPING RATES}

In Fig. 3, the calculated distributions of the damping rates $\Gamma_{n \mathbf{k}}=\tau_{n \mathbf{k}}^{-1}$ of hot electrons and holes for all the values of band index $n$ and wave vectors $\mathbf{k}$-with $\mathbf{k}$ belonging to the IBZ - used in the calculations, both using the scalar-relativistic energy spectrum (black dots) and including the SO coupling in the band structure (red dots), are presented. In addition, the results obtained using the Lindhard dielectric function ${ }^{64}$ together with the $a b$ initio scalar-relativistic wave functions and one-particle energies are shown (blue dots). The orange 
line is the result for a FEG model, i.e., when the band structure is described by a band with parabolic dispersion, the wave functions are represented by plane waves, and the Lindhard dielectric function is used for the screening. In the inset of Fig. 3, the green curve represents the Quinn-Ferrel result ${ }^{1}$ for the damping rate for very low-energy quasiparticles:

$$
\tau_{Q F}^{-1}=\frac{r_{s}^{5 / 2}\left(\varepsilon-E_{F}\right)^{2}}{263} e V^{-2} f s^{-1},
$$

where $r_{s}$ is the valence charge-density parameter of the system. For a discussion on the validity of Eq. (9) and the approximations that lead to it, see, e.g., Appendix C of Ref. 65. In the following, we shall refer to these five kinds of lifetime calculations as SC, SO, LDF-SC, FEG, and QF, respectively.

There are three main features in the distribution of the damping rates in Fig. 3. First, for $-8<\varepsilon<-6 \mathrm{eV}$, the damping rates corresponding to the $s$-like holes are presented. On the opposite side of the studied energy range, the values of $\Gamma_{n \mathbf{k}}$ for excited electrons in the $d$-like bands appear for energies in the $5.5<\varepsilon<8-\mathrm{eV}$ interval. However the main feature of Fig. 3 is the paraboliclike distribution of the damping rate for the $p$-like holes and electrons in bands crossing the Fermi level. The linewidth data for the $p$ states are separated from those for the $s$-like holes by the energy gap of $\approx 2.5 \mathrm{eV}$ presented in the $\mathrm{Pb}$ band structure.

It is interesting to compare the observed difference on the calculated damping rates for $p$ and $d$ electrons with the same energies. As can be seen in Fig. 3, at the same energy in the $5.5<\varepsilon<8-\mathrm{eV}$ interval, quasiparticles in the $d$ bands present a damping rate roughly two times smaller in comparison with that for the $p$ bands. This is a consequence of the different coupling matrices [see Eq. (3)] for $p$ and $d$ states, thereby signaling a strong localization effect in lifetimes in $\mathrm{Pb}$.

For quasiparticles in the $p$-like states, the role of final states in their decay [see Eq. (2)] can be seen in Fig. 3 from comparison of the distribution of the LDF-SC results (blue dots) with the FEG line. As in both these cases the Lindhard dielectric function was employed, the difference arises only in the wave functions entering the coupling matrix expression, Eq. (3). The blue dots show the result of the calculations using the self-consistent Kohn-Sham scalar-relativistic wave functions, whereas the orange line is obtained when wave functions are represented by the plane waves. For the $p$ electrons and holes in the energy range $-4 \leqslant \varepsilon \leqslant 5 \mathrm{eV}$, both sets of data look very similar. Nevertheless, a more careful analysis (presented below) shows some differences, which are reflected in the calculated inelastic lifetimes (see Table IV).

The strongest SO effect in the lifetime is observed for $p$ states in the vicinity of the BZ center. In Fig. 3, the arrow points to the $\Gamma(\mathrm{n}=2, \mathbf{k})$ results for the second band from the bottom, and $\mathbf{k}$ vectors close to the $\Gamma$ point calculated with the inclusion of the SO splitting in the band structure. As an example, in Table I the linewidth values for the lowest $p$ band states at some k's along the $\Gamma X$ direction are presented. Note that the same trend holds for $\mathbf{k}$ 's along the other symmetry directions as well. These data demonstrate how the giant SO splitting of the $p$ bands around the $\Gamma$ point reduces the linewidth of the excited states in this band by as much as $\approx 60 \%$, which is mainly explained by the reduction of lower energy states available for the excited electron to decay.
TABLE I. Linewidth for the lowest $p$ states at k's in vicinity of the $\Gamma$ point along the $\Gamma X$ direction, calculated at the scalar-relativistic level $\left(\Gamma^{\mathrm{sc}}\right)$ and including the SO splitting $\left(\Gamma^{\mathrm{so}}\right)$ in the band structure. All values are in $\mathrm{meV}$. The $\mathbf{k}$ vectors are labeled with the $\kappa$ index, corresponding to $\mathbf{k}=\frac{2 \pi}{24 a_{c}}(\kappa, 0,0)$.

\begin{tabular}{lrrrcccc}
\hline \hline$\kappa$ & 0 & 1 & 2 & 3 & 4 & 5 & 6 \\
\hline$\Gamma^{\mathrm{sc}}$ & 1135 & 1100 & 1010 & 895 & 776 & 663 & 559 \\
$\Gamma^{\mathrm{so}}$ & 440 & 430 & 410 & 381 & 345 & 307 & 268 \\
\hline \hline
\end{tabular}

Very recently, lifetime measurements have been performed for the thin $\mathrm{Pb}$ films with (111) orientation grown on silicon substrates. ${ }^{40,41}$ Such orientation corresponds to the quantization of bulk electronic states along the $\Gamma L$ symmetry direction. Figure 4 presents the calculated damping rates for the excited electrons in $p$ states along this direction. In contrast to the situation for the electronic states around the $\Gamma$ point, one can see that the SO interaction produces a small effect on lifetime for these states. Even if electron-electron interaction gives rise to the main contribution to the damping rates in metals, electron-phonon interaction and scattering by defects also give non-negligible contributions for low quasiparticle energies (for further discussion, see Ref. 40). Then, from Fig. 4 one can deduce that the calculated results are in reasonably good agreement with the experimental data for thin films. ${ }^{40,41}$ This signals that low-energy quasiparticles dynamics in such systems can be well represented by that in bulk $\mathrm{Pb}$. Note also how the lifetime data for states along the $\Gamma L$ direction in Fig. 4 both in the SC and SO calculations are well fitted by a quadratic function over a rather extended energy range.

\section{A. Dependence on the quasiparticle energy}

Although the damping rate $\Gamma_{n \mathbf{k}}$ of a quasiparticle in a state $(n, \mathbf{k})$ depends on its band index $n$ and on its wave vector $\mathbf{k}$,

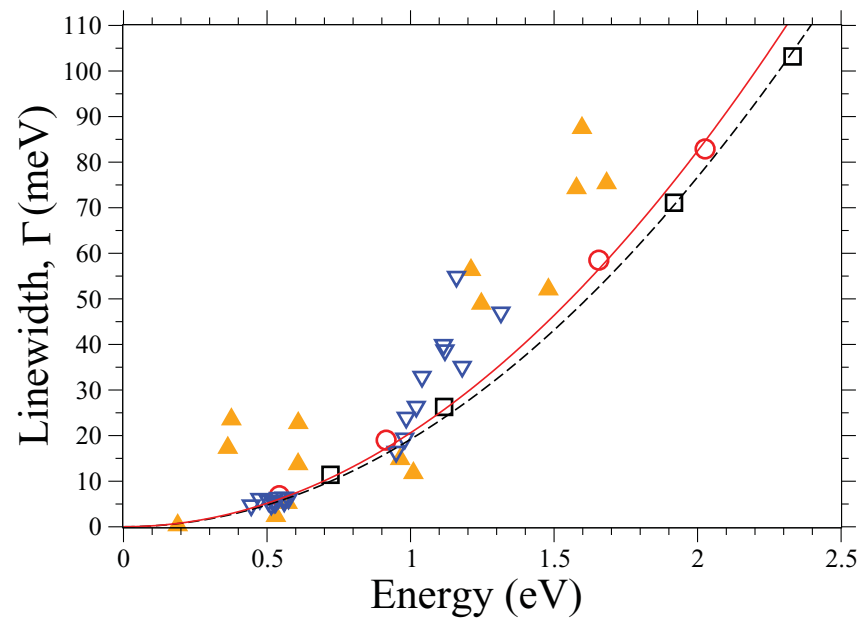

FIG. 4. (Color online) Calculated ab initio damping rates for excited electrons in states near the Fermi level with wave vectors along the $\Gamma L$ direction without (squares, dashed line) and with (circles, solid line) inclusion of the SO interaction. Lines are fits by a quadratic function, $\Gamma=\alpha \times \varepsilon^{2}$. Filled triangles and empty inverted triangles show experimental values extracted from $\mathrm{Pb}$ thin film measurements of Refs. 40 and 41, respectively. 


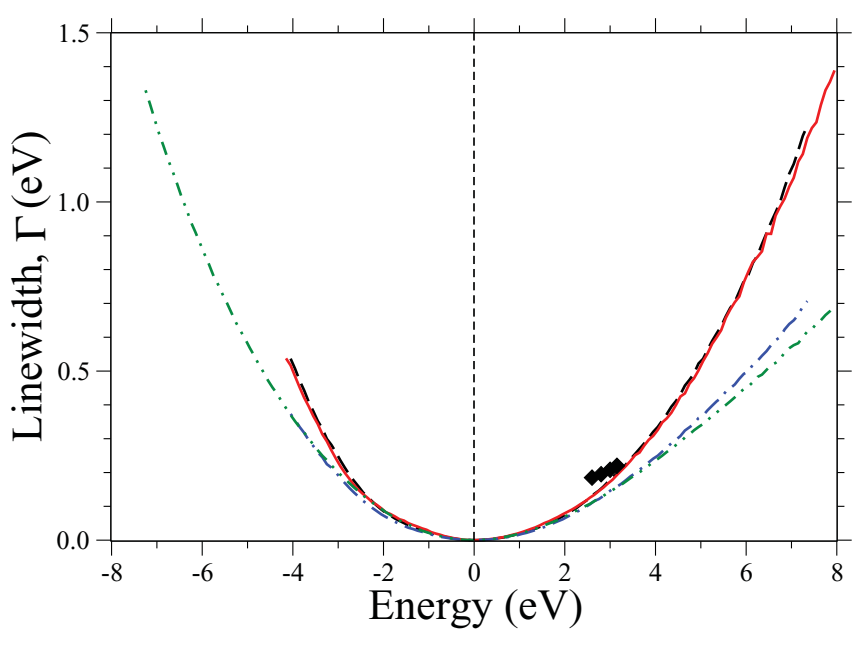

FIG. 5. (Color online) Energy dependence of averaged damping rates, $\Gamma(\varepsilon)$, of quasiparticles in the $p$-like bands obtained in the scalar-relativistic calculation (dashed line), the one including the SO interaction (solid line) and the calculation which includes the ab initio eigenstates and the Lindhard dielectric function (dashed-dotted line). The dashed-dotted-dotted line shows the same as the orange solid line in Fig. 3. Diamonds show the averaged lifetimes in bulk $\mathrm{Pb}$ measured with TR-2PPE. ${ }^{42}$

one can define $\Gamma(\varepsilon)$ as an average of $\Gamma_{n \mathbf{k}}$ over states with all wave vectors and bands lying at the same energy in the BZ. In Fig. 5, the results for $\Gamma(\varepsilon)$ are presented for quasiparticles in the $p$-like bands. The quasilinear energy dependence of the averaged damping rate for holes with energies $\varepsilon \leqslant-2.5 \mathrm{eV}$ requires a separate analysis of the dependence of $\Gamma(\varepsilon)$ for hot electrons and holes.

In Fig. 5, a different energy dependence of the averaged damping rate for holes is observed for energies below and above $\varepsilon \approx-2.5 \mathrm{eV}$. At energies $\varepsilon$ above $-2.5 \mathrm{eV}$, the averaged linewidth presents a quasiquadratic dependence on quasiparticle energy. For more detailed analysis of this dependence, we fitted the $\Gamma(\varepsilon)$ data for $-2.5 \mathrm{eV} \leqslant \varepsilon \leqslant 0$ by a third-order polynomial:

$$
\Gamma_{\text {fit }}(\varepsilon)=a_{1} \times \varepsilon+a_{2} \times \varepsilon^{2}+a_{3} \times \varepsilon^{3} .
$$

The coefficients $a_{i}$ obtained from this fitting are presented in Table II for all four kinds of linewidth calculations. Note the highest value of the curvature in the SO case, which is a direct consequence of the avoiding band-crossing effect produced by inclusion of the SO coupling in the Hamiltonian seen in Fig. 1. In the $-4.2 \leqslant \varepsilon \leqslant-2.5-\mathrm{eV}$ energy range, the coefficients $a_{1} \approx a_{2} \gg a_{3}$ for the SC-, SO-, and LDF-SC-calculated curves present a strong contribution of a linear term with the exception of the FEG results, which are well described by a quadratic function for any energy. Hence, the quasilinear behavior of the averaged damping rates in all the calculations performed with the use of the $a b$ initio eigenstates reflects the non-freeelectron-like behavior of holes in the lowest $p$ band at energies below $-2.5 \mathrm{eV}$.

In the case of electrons, all curves in Fig. 5 present an apparent quadratic dependence on the quasiparticle energy. Nevertheless, in this case we performed also the fitting procedure with the use of Eq. (10). Table II presents the obtained corresponding coefficients $a_{i}$ as well. At first sight, all four studied curves show the expected quadratic energy dependence, with the FEG results presenting the greatest deviation. At the same time, from the data of Table II it is clear that the SO curve has the strongest curvature (the biggest quadratic coefficient).

An interesting point comes from the comparison of two $\Gamma(\varepsilon)$ curves calculated using the Lindhard dielectric screening (the LDF-SC and FEG cases) with the two curves calculated using the ab initio screening (the SC and SO cases). The former ones deviate considerably from the two latter ones for energies above $\sim 3 \mathrm{eV}$. This fact cannot be explained by the difference in the curvature (i.e., the $a_{2}$ coefficients), which is similar for all four curves (see Table II) and originates from different signs of the $a_{3}$ coefficients (the sign of which being related to the screening). As a result, though $a_{2} \gg a_{3}$, for sufficiently high electron energies $(\varepsilon \geqslant \sim 3 \mathrm{eV})$ the cubic term in the dependence of $\Gamma(\varepsilon)$ on the energy can start to play a significant role.

From the results for $\Gamma(\varepsilon)$, an effective charge-density parameter $r_{s}^{\text {eff }}$ can be derived with the use of the Quinn-Ferrel expression (9). Fitting again the averaged linewidth curves with Eq. (10), new $a_{i}$ coefficients are calculated. Because of the approximations that lead to Eq. (9) (see Appendix C of Ref. 65), $\tau_{Q F}^{-1}$ is only valid for very low-energy quasiparticles. Thus, the new fitting is carried in the energy range $-1 \leqslant \varepsilon \leqslant$ $1 \mathrm{eV}$, averaging the effect of possible different curvatures for electrons and holes. Finally, the $r_{s}^{\text {eff }}$ parameters are found using the following expression:

$$
r_{s}^{\mathrm{eff}}=\left(399.7 \times a_{2}\right)^{2 / 5}
$$

In Table III, the calculated values of $r_{s}^{\text {eff }}$ and their deviation from the conventional $r_{s}=2.298$ a.u., derived from average valence density in $\mathrm{Pb}$, are presented. One can see that in this energy interval the lifetimes derived in the FEG model can closely be described by the QF expression. The effect of inclusion of the $a b$ initio band structure at the LDF-SC level consists of notable reduction of the $r_{s}^{\text {eff }}$, whereas incorporation of the screening evaluated at the $a b$ initio level works in the

TABLE II. Fitting coefficients of the $\Gamma(\varepsilon)$ data using a third-order polynomial of Eq. (10) for holes in the energy interval above $-2.5 \mathrm{eV}$

\begin{tabular}{|c|c|c|c|c|c|c|c|c|}
\hline & \multicolumn{4}{|c|}{ holes } & \multicolumn{4}{|c|}{ electrons } \\
\hline & $\mathrm{SC}$ & $\mathrm{SO}$ & LDF-SC & FEG & $\mathrm{SC}$ & $\mathrm{SO}$ & LDF-SC & FEG \\
\hline$a_{1}$ & -0.0030 & 0.0000 & -0.0020 & 0.0000 & 0.0007 & -0.0020 & 0.0002 & 0.0060 \\
\hline$a_{2}$ & 0.0195 & 0.0313 & 0.0150 & 0.0216 & 0.0175 & 0.0190 & 0.0180 & 0.0161 \\
\hline$a_{3}$ & 0.0000 & 0.0050 & -0.0009 & -0.0002 & 0.0007 & 0.0004 & -0.0007 & -0.0008 \\
\hline
\end{tabular}
and $p$ electrons. Meaning of abbreviations "SC", "SO", "LDF-SC", and "FEG" is explained in the text. $a_{2}$ in $\mathrm{eV}^{-1}$ and $a_{3}$ in $\mathrm{eV}^{-2}$. 
TABLE III. Effective charge-density parameters obtained from Eq. (11) on base of four sets of data as explained in the text. $\delta r_{s}$ stands for the deviation from a conventional value for $\mathrm{Pb} r_{s}=$ 2.298 a.u.

\begin{tabular}{lcccc}
\hline \hline & SC & SO & LDF-SC & FEG \\
\hline$r_{s}^{\text {eff }}$ & 2.37 & 2.49 & 2.18 & 2.33 \\
$\delta r_{s}^{\text {eff }}(\%)$ & +3.0 & +8.3 & -5.2 & +1.3 \\
\hline \hline
\end{tabular}

opposite direction, increasing $r_{s}^{\text {eff }}$. Also, a higher value of $r_{s}^{\text {eff }}$ in the $\mathrm{SO}$ case in comparison with the $\mathrm{SC}$ one signals a stronger effective screening in the former case at such low energies.

In Table IV, we present the calculated values for averaged inelastic lifetimes for excited electrons and holes in $\mathrm{Pb}$ at $|\varepsilon|=0.5,1.0,2.0$, and $3.0 \mathrm{eV}$ obtained in all the calculations. For excited electrons, the QF, FEG, and SC calculations give similar results. Noting that the calculated lifetimes using the self-consistent eigenstates and the Lindhard screening differ from the other three ones, we can conclude that, even if lead is a free-electron-like metal, the lifetime of quasiparticles in bulk $\mathrm{Pb}$ is the result of a balance between screening and localization. Interestingly, the lifetimes in this energy interval calculated including the SO splitting in the band structure are the lowest ones for each quasiparticle energy, both for electrons and holes.

As seen in Fig. 1, at the $W$ point only one unoccupied $p$ band approaches but does not cross $E_{F}$, presenting a local minimum. This proximity to the Fermi level together with the absence of available unoccupied states for decay with small momentum transfers leads to notably longer inelastic lifetimes for the states around the $W$ point. This is a strong band-structure effect. In Fig. 6, we compare the averaged inelastic lifetimes $\tau^{\text {av }}$ of electrons with very low energies (solid line) with the values calculated for states close to $W$ (shown by symbols) obtained in the scalar-relativistic calculation (note that for this analysis the SO interaction has a negligible effect). For comparison, the FEG results are shown by a dashed line. As can be seen, upon approaching the $W$ point (reducing energy) $\tau$ increases faster in comparison with the averaged inelastic lifetime at those energies, being $\approx 50 \%$ higher than $\tau^{\text {av }}$ at the same energy in other parts of the BZ. Hence, around the $W$ point, $\tau$ behaves in a non-free-electron-like manner.

\section{B. Ratio of the lifetime of electrons and holes}

In Fig. 7, the ratios of lifetimes for electrons $\tau_{e}$ and holes $\tau_{h}$ at the same absolute value of the quasiparticle energy are shown for four different calculations. The curves show two energy ranges with different behaviors. At energies $|\varepsilon| \geqslant 2 \mathrm{eV}$,

TABLE IV. Lifetime of excited electrons (holes) at four different values of $|\varepsilon|$. All values are in fs.

\begin{tabular}{lrrrrr}
\hline \hline$|\varepsilon|$ & \multicolumn{1}{c}{ SC } & SO & LDF-SC & FEG & QF \\
\hline $0.5 \mathrm{eV}$ & $130(114)$ & $114(87)$ & $162(145)$ & $128(130)$ & 133 \\
$1.0 \mathrm{eV}$ & $34(29)$ & $31(23)$ & $41(36)$ & $34(31)$ & 33 \\
$2.0 \mathrm{eV}$ & $9(8)$ & $8(7)$ & $10(9)$ & $9(7)$ & 8 \\
$3.0 \mathrm{eV}$ & $4(3)$ & $4(3)$ & $4(3)$ & $5(3)$ & 4 \\
\hline \hline
\end{tabular}

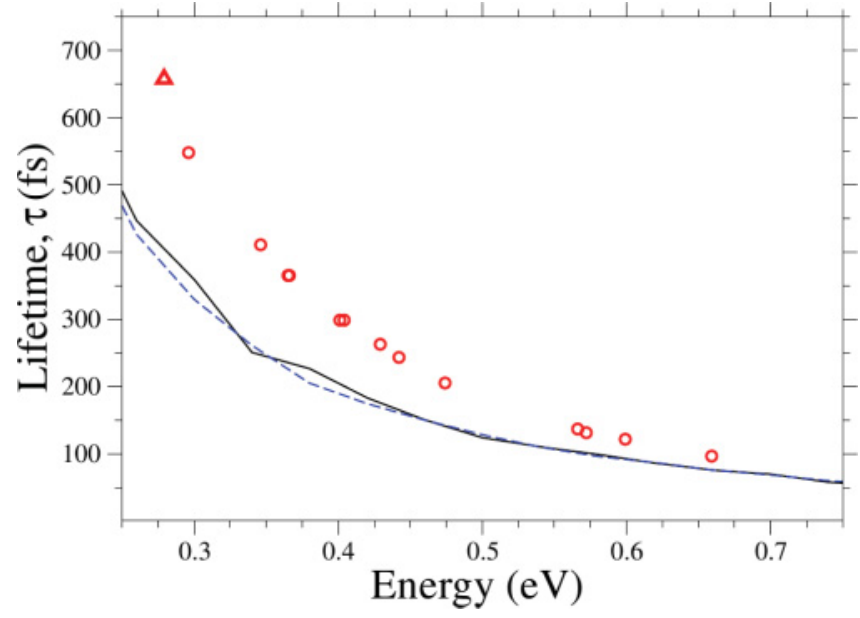

FIG. 6. (Color online) Averaged inelastic lifetimes of electrons (solid line) and the lifetimes calculated for states at k's in vicinity of the $W$ point (circles) evaluated in the scalar-relativistic calculation. Triangle highlights $\tau$ at the W point. Dashed line: FEG results.

all the four curves present a quasilinear behavior. However, for quasiparticle energies below $2 \mathrm{eV}$, only the FEG curve remains quasilinear. The other three calculations present quite different dependencies of the $\tau_{e} / \tau_{h}$ ratio at those energies. The quasilinear behavior of $\tau_{e} / \tau_{h}$ is found in the homogeneous electron-gas calculations (see, e.g., Fig. 2 of Ref. 65). Hence, in bulk $\mathrm{Pb}$ the band-structure effects are important in the electronelectron inelastic scattering processes for quasiparticles with energies less than $2 \mathrm{eV}$. As the LDF-SC curve deduced from the lifetime results obtained with the use the Lindhard screening and the $a b$ initio eigenstates presents also the band-structure effects, these effects are the consequence of using the true eigenstates in the evaluation of the coupling matrices [see Eq. (3)] and not of the ab initio screening used. Note also that $\tau_{e}>\tau_{h}$, for all energies in all four levels of calculations.

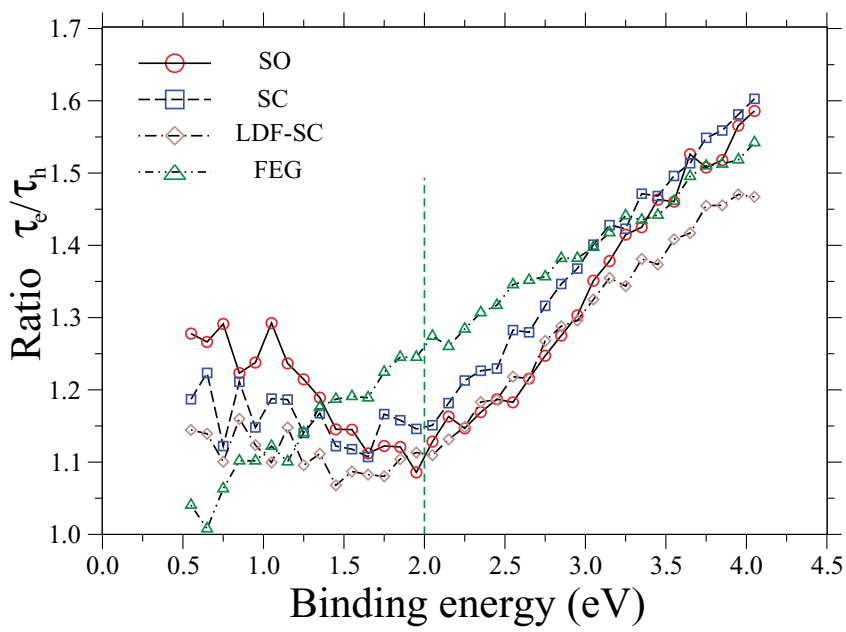

FIG. 7. (Color online) Ratio $\tau_{e}(\varepsilon) / \tau_{h}(\varepsilon)$ as a function of the quasiparticle energy $\varepsilon$ for four levels of calculations. The dash line separates two energy regions with different physical behavior (see text). Lines are guides to the eye. 


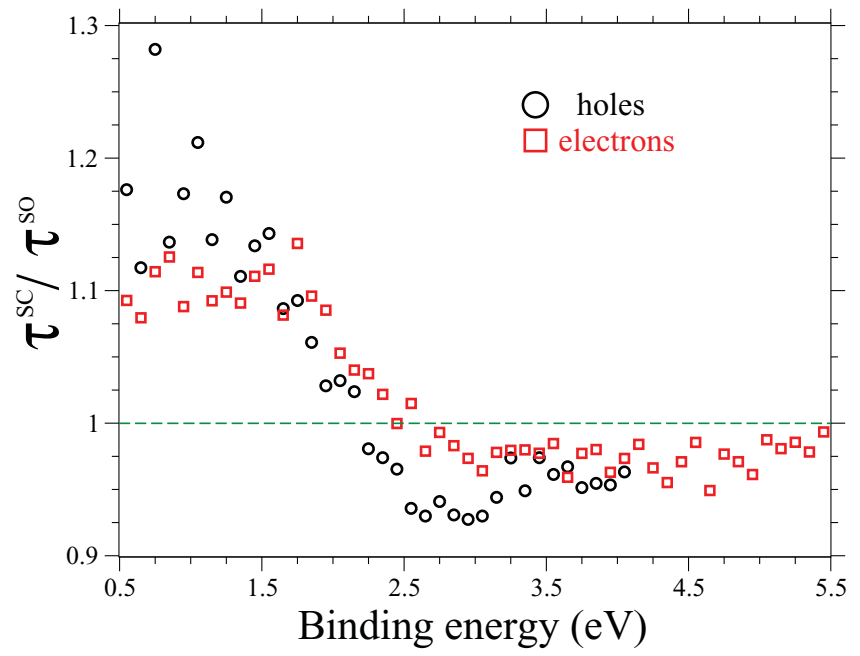

FIG. 8. (Color online) Ratio $\tau^{\mathrm{SC}}(\varepsilon) / \tau^{\mathrm{SO}}(\varepsilon)$ as a function of the quasiparticle energy $\varepsilon$ for $p$-like states, both for holes (circles) and electrons (squares).

\section{Effect of SO interaction on lifetime}

Modifications in the $\mathrm{Pb}$ band structure upon inclusion of the SO interaction increase the lifetime of electrons in the $d$ bands and reduce that for holes in the $s$ ones, as demonstrated in Fig. 3. In all the cases, the variation in $\tau$ upon inclusion of the SO term does not exceed $10 \%$ in comparison with values obtained in the scalar-relativistic calculations. In Fig. 8, the ratio $\left.\tau\left(\varepsilon^{\mathrm{SC}}\right) / \tau_{(} \varepsilon^{\mathrm{SO}}\right)$ as a function of energy is plotted for the $p$ holes and electrons. For holes with $\varepsilon \leqslant-2 \mathrm{eV}$ and electrons with $2.5 \leqslant \varepsilon \leqslant 5.5 \mathrm{eV}$, inclusion of the $\mathrm{SO}$ splitting increases the quasiparticle lifetimes, in both cases less than $10 \%$. On the other hand, for holes with binding energies less than $2 \mathrm{eV}$, spin-orbit-induced splitting reduces lifetimes by as much as $30 \%$. For electrons with $\varepsilon \leqslant 2.5 \mathrm{eV}$, as can be seen in Fig. 8, the SO interaction lowers $\tau$ by $\approx 10 \%$. One can expect that this tendency will continue for electronic states around the Fermi surface.

\section{CONCLUSIONS}

We have presented full GW-RPA calculations of the inelastic damping rates of low-energy quasiparticles in bulk $\mathrm{Pb}$ and studied in detail the band-structure effects as well as the consequences of the inclusion of the spin-orbit interaction. A strong localization effect is found for electrons with energies $5.5 \mathrm{eV} \leqslant \varepsilon \leqslant 8 \mathrm{eV}$, where lifetime for $d$ states is almost twice that of $p$ states.

The states in the lowest valence $p$ energy band at the center of the BZ reduce their damping rates by roughly $60 \%$ upon inclusion of the spin-orbit interaction in comparison with the pure scalar-relativistic calculation. For the $p$ electrons, the damping rates $\Gamma(\varepsilon)$ averaged over the BZ present a quadratic dependence on energy $\varepsilon$, whereas for $p$ holes the quasilinear dependence of $\Gamma(\varepsilon)$ for energies $\varepsilon$ below $-2.5 \mathrm{eV}$ reflects a non-free-electron-like nature of the electronic states at the bottom of the lowest $p$ band. The calculated lifetime ratio $\tau_{e}(\varepsilon) / \tau_{h}(\varepsilon)$ reveals that band-structure effects are important in electron-electron inelastic scattering processes for quasiparticles with binding energies less than $2 \mathrm{eV}$. Present lifetime calculations for bulk $\mathrm{Pb}$ are in good agreement with inelastic damping rates of quantum well states of metallic thin films measured recently, thereby presenting evidence that quasiparticle dynamics can be considered as being bulklike even in very thin $\mathrm{Pb}$ films.

Finally, one can think of new experiments that could test the effect of the physical ingredients studied in the present work. As an example, studies of field emission resonances on $\mathrm{Pb}(111)^{66-68}$ could shed light on spin-orbit effects analyzed in the present work. Band-structure effects may be tested too by experimental studies on $\mathrm{Pb}$ films grown in different crystallographic directions.

\section{ACKNOWLEDGMENTS}

We acknowledge financial support from the Spanish Ministerio de Ciencia e Innovación (Grant No. FIS2010-19609-C0201), the Departamento de Educación del Gobierno Vasco, and the University of the Basque Country (Grant No. GIC07-IT366-07).
${ }^{1}$ J. J. Quinn and R. A. Ferrell, Phys. Rev. 112, 812 (1958).

${ }^{2}$ P. Nozières, Theory of Interacting Fermi Liquids (Benjamin, New York, 1962).

${ }^{3}$ I. A. Nechaev and E. V. Chulkov, Phys. Rev. B 73, 165112 (2006).

${ }^{4}$ D. R. Penn, S. P. Apell, and S. M. Girvin, Phys. Rev. B 32, 7753 (1985).

${ }^{5}$ E. Zarate, P. Apell, and P. M. Echenique, Phys. Rev. B 60, 2326 (1999).

${ }^{6}$ V. P. Zhukov and E. V. Chulkov, Phys. Usp. 52, 105 (2009); Usp. Fiz. Nauk 179, 113 (2009).

${ }^{7}$ I. Campillo, J. M. Pitarke, A. Rubio, E. Zarate, and P. M. Echenique, Phys. Rev. Lett. 83, 2230 (1999).

${ }^{8}$ W.-D. Schöne, R. Keyling, M. Bandić, and W. Ekardt, Phys. Rev. B 60, 8616 (1999).

${ }^{9}$ R. Keyling, W.-D. Schöne, and W. Ekardt, Phys. Rev. B 61, 1670 (2000).
${ }^{10}$ F. Ladstädter, U. Hohenester, P. Puschnig, and C. Ambrosch-Draxl, Phys. Rev. B 70, 235125 (2004).

${ }^{11}$ E. V. Chulkov, A. G. Borisov, J. P. Gauyacq, D. Sánchez-Portal, V. M. Silkin, V. P. Zhukov, and P. M. Echenique, Chem. Rev. 106, 4160 (2006).

${ }^{12}$ I. A. Nechaev, V. P. Zhukov, and E. V. Chulkov, Phys. Solid State 49, 1811 (2007) [Fiz. Tverd. Tela (St.-Petersburg) 49, 1729 (2007)].

${ }^{13}$ R. Diez Muino, D. Sanchez-Portal, V. M. Silkin, E. V. Chulkov, and P. M. Echenique, PNAS 108, 971 (2011).

${ }^{14}$ I. Campillo, V. M. Silkin, J. M. Pitarke, E. V. Chulkov, A. Rubio, and P. M. Echenique, Phys. Rev. B 61, 13484 (2000).

${ }^{15}$ I. A. Nechaev, I. Yu. Sklyadneva, V. M. Silkin, P. M. Echenique, and E. V. Chulkov, Phys. Rev. B 78, 085113 (2008).

${ }^{16}$ V. M. Silkin, E. V. Chulkov, and P. M. Echenique, Phys. Rev. B 68, 205106 (2003) 
${ }^{17}$ W. Ekardt, W.-D. Schöne, and R. Keyling, Appl. Phys. A 71, 529 (2000).

${ }^{18}$ W.-D. Schöne, Int. J. Mod. Phys. B 17, 5655 (2003).

${ }^{19}$ I. G. Gurtubay, J. M. Pitarke, and P. M. Echenique, Phys. Rev. B 69, 245106 (2004).

${ }^{20}$ V. P. Zhukov, F. Aryasetiawan, E. V. Chulkov, I. G. de Gurtubay, and P. M. Echenique, Phys. Rev. B 64, 195122 (2001).

${ }^{21}$ M. R. Bacelar, W.-D. Schöne, R. Keyling, and W. Ekardt, Phys. Rev. B 66, 153101 (2002).

${ }^{22}$ W.-D. Schöne, Prog. Surf. Sci. 82, 161 (2007).

${ }^{23}$ V. P. Zhukov, E. V. Chulkov, and P. M. Echenique, Phys. Rev. Lett. 93, 096401 (2004).

${ }^{24}$ I. A. Nechaev and E. V. Chulkov, Phys. Solid State 51, 754 (2009)

[Fiz. Tverd. Tela (St.-Petersburg) 51, 713 (2009)].

${ }^{25}$ I. A. Nechaev and E. V. Chulkov, Eur. Phys. J. B 77, 31 (2010).

${ }^{26}$ L. Hedin and S. Lundqvist, Solid State Phys. 23, 1 (1969).

${ }^{27}$ A. L. Fetter and J. D. Walecka, Quantum Theory of Many-Particle Systems (McGraw-Hill, Boston, 1971).

${ }^{28}$ G. D. Mahan, Many-Particle Physics (Plenum, New York, 1990).

${ }^{29}$ V. P. Zhukov, O. Andreyev, D. Hoffmann, M. Bauer, M. Aeschlimann, E. V. Chulkov, and P. M. Echenique, Phys. Rev. B 70, 233106 (2004).

${ }^{30}$ V. P. Zhukov, E. V. Chulkov, and P. M. Echenique, Phys. Rev. B 72, 155109 (2005).

${ }^{31}$ A. B. Schmidt, M. Pickel, M. Donath, P. Buczek, A. Ernst, V. P. Zhukov, P. M. Echenique, L. M. Sandratskii, E. V. Chulkov, and M. Weinelt, Phys. Rev. Lett. 105, 197401 (2010).

${ }^{32}$ V. P. Zhukov, F. Aryasetiawan, E. V. Chulkov, and P. M. Echenique, Phys. Rev. B 65, 115116 (2002).

${ }^{33}$ A. Marini, R. Del Sole, A. Rubio, and G. Onida, Phys. Rev. B 66, 161104 (2002).

${ }^{34}$ Z. Yi, Y. Ma, M. Rohlfing, V. M. Silkin, and E. V. Chulkov, Phys. Rev. B 81, 125125 (2010).

${ }^{35}$ Y. Guo et al., Science 306, 1915 (2004).

${ }^{36}$ D. Eom, S. Qin, M.-Y. Chou, and C. K. Shih, Phys. Rev. Lett. 96, 027005 (2006).

${ }^{37}$ S. Qin, J. Kim, Q. Niu, and C.-K. Shih, Science 324, 1314 (2009).

${ }^{38}$ C. Brun, I.-P. Hong, F. Pattley, I. Y. Sklyadneva, R. Heid, P. M. Echenique, K. P. Bohnen, E. V. Chulkov, and W.-D. Schneider, Phys. Rev. Lett. 102, 207002 (2009).

${ }^{39}$ T. Zhang, P. Cheng, W.-J. Li, Y.-J. Sun, G. Wang, X.-G. Zhu, K. He, L. Wang, X. Ma, and X. Chen, Nat. Phys. 6, 104 (2010).

${ }^{40}$ I.-P. Hong et al., Phys. Rev. B 80, 081409(R) (2009).

${ }^{41}$ P. S. Kirchmann, L. Rettig, X. Zubizarreta, V. M. Silkin, E. V. Chulkov, and U. Bovensiepen, Nat. Phys. 6, 782 (2010).
${ }^{42}$ S. Mathias, A. Ruffing, F. Deicke, M. Wiesenmayer, M. Aeschlimann, and M. Bauer, Phys. Rev. B 81, 155429 (2010).

${ }^{43}$ P. S. Kirchmann and U. Bovensiepen, Phys. Rev. B 78, 035437 (2008).

${ }^{44}$ M. Becker and R. Berndt, Phys. Rev. B 81, 205438 (2010).

${ }^{45}$ S. M. Lu, W. B. Su, C. L. Lin, W. Y. Chan, H. L. Hsiao, C. S. Chang, and T. T. Tsong, J. Appl. Phys. 108, 083707 (2010).

${ }^{46}$ P. Hohenberg and W. Kohn, Phys. Rev. 136, B864 (1964).

${ }^{47}$ W. Kohn and L. Sham, Phys. Rev. 140, A1133 (1965).

${ }^{48}$ E. V. Chulkov, V. M. Silkin, and M. Machado, Surf. Sci. 482, 693 (2001).

${ }^{49}$ S. Link, H. A. Dürr, G. Bihlmayer, S. Blügel, W. Eberhardt, E. V. Chulkov, V. M. Silkin, and P. M. Echenique, Phys. Rev. B 63, 115420 (2001).

${ }^{50}$ E. V. Chulkov, J. Kliewer, R. Berndt, V. M. Silkin, B. Hellsing, S. Crampin, and P. M. Echenique, Phys. Rev. B 68, 195422 (2003).

${ }^{51}$ C. Corriol, V. M. Silkin, D. Sánchez-Portal, A. Arnau, E. V. Chulkov, P. M. Echenique, T. von Hofe, J. Kliewer, J. Kröger, and R. Berndt, Phys. Rev. Lett. 95, 176802 (2005).

${ }^{52}$ E. Runge and E. K. U. Gross, Phys. Rev. Lett. 52, 997 (1984).

${ }^{53}$ M. Petersilka, U. J. Gossmann, and E. K. U. Gross, Phys. Rev. Lett. 76, 1212 (1996).

${ }^{54}$ S. L. Adler, Phys. Rev. 126, 413 (1962).

${ }^{55}$ N. Troullier and J. L. Martins, Phys. Rev. B 43, 1993 (1991).

${ }^{56}$ D. M. Ceperley and B. J. Alder, Phys. Rev. Lett. 45, 566 (1980).

${ }^{57}$ J. P. Perdew and A. Zunger, Phys. Rev. B 23, 5048 (1981).

${ }^{58}$ F. Aryasetiawan and O. Gunnarsson, Phys. Rev. B 49, 16214 (1994).

${ }^{59}$ V. M. Silkin, I. P. Chernov, Yu. M. Koroteev, and E. V. Chulkov, Phys. Rev. B 80, 245114 (2009).

${ }^{60} \mathrm{M}$. Tinkam, Group Theory and Quantum Mechanics (McGraw-Hill, New York, 1971).

${ }^{61}$ M. J. Verstraete, M. Torrent, F. Jollet, G. Zérah, and X. Gonze, Phys. Rev. B 78, 045119 (2008).

${ }^{62}$ G. Jézéquel and I. Pollini, Phys. Rev. B 41, 1327 (1990).

${ }^{63}$ E. V. Chulkov, Y. M. Koroteev, and V. M. Silkin, Surf. Sci. 247, 115 (1991).

${ }^{64} \mathrm{D}$. Pines and P. Nozières, The Theory of Quantum Liquids (Benjamin, New York, 1966).

${ }^{65}$ P. M. Echenique, J. M. Pitarke, E. V. Chulkov, and A. Rubio, Chem. Phys. 251, 1 (2000).

${ }^{66}$ A. Zugarramurdi, N. Zabala, V. M. Silkin, A. G. Borisov, and E. V. Chulkov, Phys. Rev. B 80, 115425 (2009).

${ }^{67}$ M. C. Yang, C. L. Lin, W. B. Su, S. P. Lin, S. M. Lu, H. Y. Lin, C. S. Chang, W. K. Hsu, and T. T. Tsong, Phys. Rev. Lett. 102, 196102 (2009).

${ }^{68}$ A. Zugarramurdi, N. Zabala, A. G. Borisov, and E. V. Chulkov, Phys. Rev. Lett. 106, 249601 (2011). 